\title{
Resistencia química del hormigón XXVII. Contribución al estudio del sistema: cemento P-550-ARI hidratado-agua desionizada
}

M. ${ }^{a}$ FRANCISCA BERMEJO-MUÑOZ; JOSE LUIS SAGRERA-MORENO y DEMETRIO GASPAR-TEBAR IETCC/CSIC

\section{RESUMEN}

En el trabajo presente, continuación de otros, se estudia el comportamiento de un cemento portland de alta resis tencia inicial (P-550-ARI) - con un contenido calculado (Bogue) del $39,2 \%$ de $C_{3} S$, del $25,7 \%$ de $C_{2} S$, del $9,9 \%$ de $\mathrm{C}_{3} \mathrm{~A}$, del 12,0 de $\mathrm{C}_{4} \mathrm{AF}$ y del $6,9 \%$ de $\mathrm{CaSO}_{4}$ - cuando se somete a la acción del agua desionizada que atraviesa los lechos de cemento granulados, fabricados con dicho cemento hidratado y curado durante 7 y 28 dias, determinando:

a) La evolución del contenido de los iones Ca (II) y $\mathrm{SO}_{4}$ (II) de las diversas fracciones recogidas de agua desionizada, que han atravesado los lechos mencionados, así como la del $\mathrm{pH}$ y de la conductividad,

b) la variación de las cantidades de dichos iones, que se encuentran formando los compuestos correspondientes en el cemento hidratado de los lechos antes y después de someterlos a la acción del agua desionizada, $y$

c) las modificaciones estructurales experimentadas por los compuestos cristalinos del cemento hidratado de los lechos sometidos a la acción del agua desionizada.

\section{SUMMARY}

In this work, which is the following of others, the performance of a portland cement of a high initial resistance (P-550-ARI) - with a calculated content (Bogue) of $39,2 \%$ of $C_{3} S$, of $25,7 \%$ of $C_{2} S$, of $9,9 \%$ of $C_{3} A$, of $12,0 \%$ of $\mathrm{C}_{4} \mathrm{AF}$ and of $6,9 \%$ of $\mathrm{CaSO}_{4}$ - is studied when it is submitted to the action of deionized water running across the beds of granulated cement, made with this hydrated cement cured during 7 or 28 days, determining:

a) the evolution of the content on ions $\mathrm{Ca}$ (II) and $\mathrm{SO}_{4}$ (II) on the taken diverse fractions of deionized water which have ran across those beds, so as this of the $\mathrm{pH}$ and of the conductivity,

b) the variation in the amount of these ions that are forming the correspondent compounds, in the hydrated cement from the beds that have been submitted before and after to the action of the deionized water, and

c) the structural modification undergone by the crystalline compounds of the hydrated cement from the beds submitted to the action of the deionized water.

\section{INTRODUCCION}

En trabajos anteriores se ha estudiado el comportamiento de un cemento portland de altas resistencias inciales, análogo al presente (cemento $1<>$ P-550 ARI) cuando las probetas de mortero (1:3), fabricadas con este cemento, se someten a la acción del agua potable filtrada, del agua de mar artificial y de una disolución saturada de yeso en donde han estado sumergidas durante períodos variables de tiempo $(56,90,180$ y 360 días, en estos trabajos y, posteriormente, hasta 3 años) (1) (2) (3) (4) (5) (6) y cuando han pasado 14 I de agua de mar artificial ASTM D 1141-75 a través de un lecho de mortero (1:3) (7), habiendo determinado la variación de las resistencias mecánicas a flexotracción de las probetas de $1 \times 1 \times 6 \mathrm{~cm}$, la evolución de los coeficientes de corrosión Koch-Steinegger, la composición estructural de las nuevas fases sólidas formadas en los medios en donde han estado las probetas mencionadas y de la fracción enri- quecida (pasta de cemento) extraída de las probetas de mortero y del lecho, así como la concentración de los iones $\mathrm{Ca}$ (II), $\mathrm{Mg}$ (II), $\mathrm{SO}_{4}$ (II) y $\mathrm{Cl}$ (I) de las disoluciones en donde se han conservado-atacado las probetas de mortero o que han atravesado el lecho, de las fases sólidas formadas y de la fracción extraida del lecho.

Este trabajo, continuación de otros (8) (9) (10), se ha realizado con objeto de contribuir al estudio de la resistencia química de los diversos tipos de cemento, en general, frente a la acción de aguas y disoluciones de sales agresivas y en este caso de agua desionizada, de disoluciones acuosas de sulfato de sodio y de sulfato de magnesio cuando fluyen a traves de un lecho de cemento portland de alta resistencia inicial hidratado. De los resultados obtenidos con el agua desionizada se da cuenta en este artículo y con las otras dos disoluciones agresivas se dará en sucesivas publicaciones. 


\section{PARTE EXPERIMENTAL}

En el presente trabajo, se estudia el comportamiento de un cemento portland de alta resistencia inicial (P-550-ARI), designado como cemento 1, cuando los lechos fabricados con dicho cemento 1 - una vez que se ha hidratado, granulado y conservado durante 7 ó 28 días en un recinto estanco con una humedad relativa superior al $80 \%$, a una temperatura de $21 \pm 2^{\circ} \mathrm{C}-$ se someten a la acción del agua potable desionizada (sistema: cemento 1 hidratado-agua desionizada), objeto del presente artículo, de una disolución de sulfato de sodio (sistema: cemento 1 hidratado-disolución de sulfato de sodio) $\left(^{(}\right)$y de una disolución de sulfato de magnesio (sistema: cemento 1 hidratado-disolución de sulfato de magnesio) $\left(^{*}\right)$ determinado:

a) La variación de la concentración de los iones $\mathrm{Ca}$ (II) y $\mathrm{SO}_{4}$ (II) en los dos primeros sistemas y de los iones $\mathrm{Ca}$ (II), $\mathrm{Mg}$ (II) y $\mathrm{SO}_{4}$ (II), en el tercer sistema, tanto de las disoluciones agresivas como del cemento hidratado del lecho.

b) La evolución del $\mathrm{pH}$ y de la conductividad de las fracciones extraídas de las disoluciones agresivas.

c) Las modificaciones estructurales experimentadas por los compuestos cristalinos del cemento hidratado del lecho, una vez sometido a la acción de dichas disoluciones agresivas, con relación a la composición estructural del cemento hidratado.

\subsection{Materiales utilizadas}

\section{a) Cemento 1}

Se ha utilizado un cemento portland industrial de alta resistencia inicial, cemento $1<>P$. 550-ARI, con un contenido calculado (Bogue) del $39,2 \%$ de $\mathrm{C}_{3} \mathrm{~S}$, del $25,7 \%$ de $\mathrm{C}_{2} \mathrm{~S}$, del $9,9 \%$ de $\mathrm{C}_{3} \mathrm{~A}$, del $12 \%$ de $\mathrm{C}_{4} \mathrm{AF}$ y del $6,9 \%$ de $\mathrm{CaSO}_{4}$, cuyas características, físicas, mecánicas y estructurales se señalan en (1), así como su resistencia química (Koch-Steinegger) frente al agua de mar artificial y a una disolución saturada de yeso (1) y (3).

\section{b) Disolución agresiva}

El agua desionizada, que se ha utilizado en el primer sistema, se ha obtenido a partir de agua potable, haciéndola pasar por un filtro

(*) De los resultados correspondientes a los sistemas cemento 1 hidratado-disolución de sulfato de sodio y cemento 1 hidratado-disolución de sulfato de magnesio se dará cuenta en otros trabajos. tipo Chamberlain y por un lecho mixto formado por dos resinas (catiónica sulfónica fuerte y aniónica fuerte).

\subsection{Técnica operatoria}

\section{a) Preparación del lecho}

A partir del cemento $1<>$ P-550-ARI, se ha preparado el lecho de cemento hidratado del modo siguiente:

Una vez amasado el cemento con el agua correspondiente a su consistencia normal (500 gramos de cemento y $135 \mathrm{ml}$ de agua potable filtrada) se introduce en una cámara húmeda, con una humedad relativa superior al $90 \%$ y a una temperatura de $21 \pm 2^{\circ} \mathrm{C}$. Después de ocho horas de permanencia en dicha cámara, se pasa toda la pasta de cemento por un tamiz que tiene $1,5 \mathrm{~mm}$ de luz de malla; de este modo se tiene una pasta granulada del cemento hidratado, que se lleva, a continuación, a la cámara húmeda en donde se deja siete o veintiocho días, según la edad que se considere en cada caso.

\section{b) Equipo y procedimiento operatorio}

\section{b.1. Equipo de trabajo}

El equipo de trabajo utilizado (figura 1) consta de las siguientes partes:

- depósito nodriza con una capacidad de 18 litros, en donde se coloca el agua desionizada,

- columna de plástico, en donde se sitúa el lecho granulado de la pasta de cemento, de $4 \mathrm{~cm}$ de diámetro exterior, $3,5 \mathrm{~cm}$ de diámetro interior y $16 \mathrm{~cm}$ de altura,

- tres llaves de paso, una de tres vías y las restantes de dos vías,

- Kitasatos para recoger las diversas fracciones.

\section{b.2. Procedimiento operatorio}

Se toman, aproximadamente, $70 \mathrm{~g}$ de la pasta granulada de cemento y se introducen en la columna de plástico (columna de intercambio), a través de la cual pasa el agua desionizada, recogiéndose las diversas fracciones, en función del tiempo, que se señalan en el apartado 3.

Tanto en la parte superior de los lechos (es- 
pacio que no está ocupado por el agua desionizada), como en el resto del equipo de trabajo se introduce nitrógeno, antes de comenzar el ensayo, con el fin de evitar el contacto de la pasta de cemento con el aire y, de un modo especial, la posible reacción de carbonatación.

\section{c) Determinaciones efectuadas}

En cada una de las distintas fracciones recogidas del agua desionizada, que ha atravesado el lecho, se ha determinado:

c.1. el contenido de los iones $\mathrm{Ca}$ (II) y $\mathrm{SO}_{4}$ (II); el primero por complexometria con EDTA y el segundo gravimétricamente, al estado de $\mathrm{BaSO}_{4}$,

c.2. el valor del $\mathrm{pH}$ y de la conductividad, utilizando un $\mathrm{pH}$-metro digital, Crison 501, con electrodo Metrohm AG, tipo $9100 \mathrm{He}-$ risau y un conductímetro digital, Crison 522, con célula Metrohm CH, tipo 9100 Herisau.

En la pasta granulada de cemento hidratado, antes y después de pasar el agua desionizada,

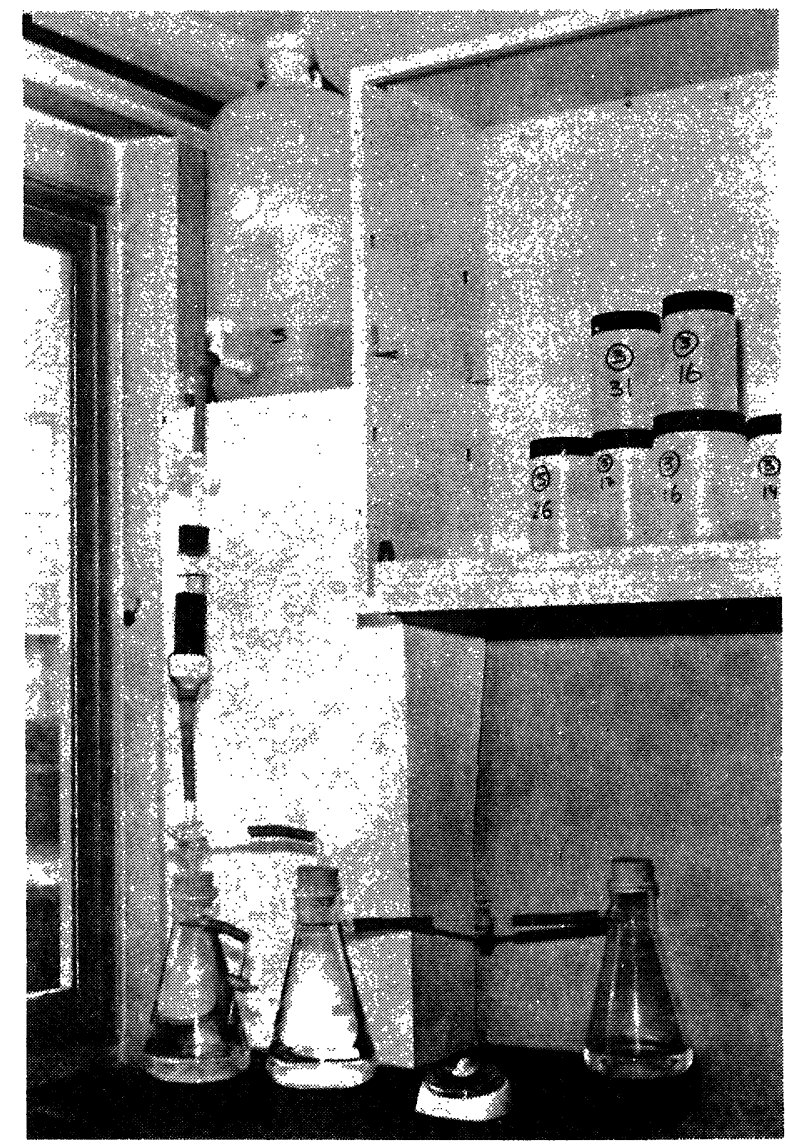

Foto: J. J. López del Amor. IETCC/CSIC.

Fig. 1.-Equipo de trabajo. se ha determinado:

c.3. el contenido de $\mathrm{Ca}$ (II) y de $\mathrm{SO}_{4}$ (II), de acuerdo con los procedimientos operatorios descritos en el Pliego RC-75 (11),

c.4. la composición estructural por DRX, utilizando el equipo Philips PW-1010 y las condiciones de trabajo que se reseñan en (9).

\section{RESULTADOS}

\section{Sistema: cemento P-550-ARI-agua desionizada}

\subsection{Volúmenes extraídos de agua desio- nizada, en función del tiempo}

En la figura 2 se incluyen los volúmenes parciales y totales, recogidos de agua desionizada, que ha pasado a través del lecho granulado de cemento, en función del tiempo, correspondientes al cemento granulado conservado 7 días en una cámara húmeda (a $21 \pm$ $2^{\circ} \mathrm{C}$ y con una humedad relativa superior al 90 $\%)$. Durante las primeras 8 horas las fracciones de agua desionizada se han recogido cada hora; el caudal que ha atravesado el lecho está comprendida entre 0,14 y $0,20 \mathrm{l} / \mathrm{h}$ y el volumen total ha sido $1,40 \mathrm{I}$. En las siguientes 15 horas, se ha hecho sólo una extracción, habiéndose recogido 2,57 I. A partir de las primeras 24 horas y hasta la extracción 22 $(120 \mathrm{~h})$, la cadencia de recogida ha sido cada 3 horas para tres fracciones ( 9 horas) y 1 en las otras 15 horas, durante 24 horas; a partir de este momento (120 horas) y hasta el final del ensayo (39 días) se ha efectuado una extracción cada 24 horas o cada 48 horas.

El volumen total de agua desionizada que ha atravesado el lecho ha sido 144,4 litros durante 39 días. Así mismo, en la figura 2 también se incluyen los volúmenes parciales y to-

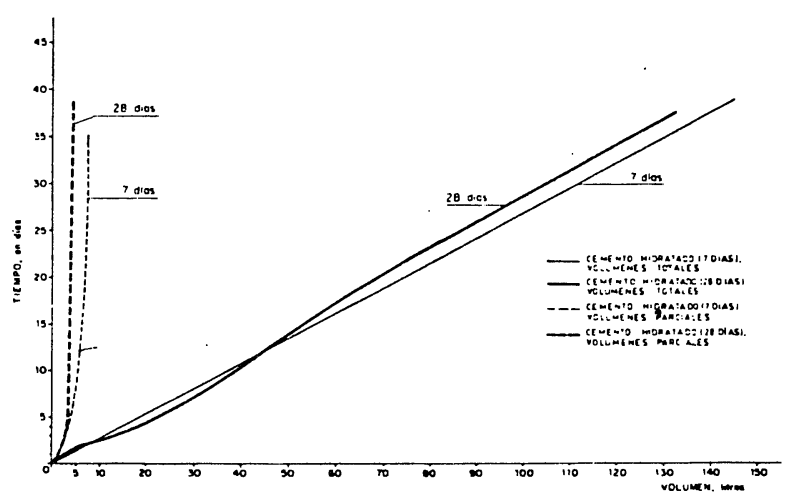

Fig. 2.-Sistemas: cemento 1 hidratado (7 y 28 días)-agua desionizada. Volúmenes parciales y totales en función del tiempo. 
tales recogidos de agua desionizada que ha pasado a través del lecho granulado de cemento 1 , hidratado y curado durante 28 dias. Durante las primeras 7 horas se han efectuado otras tantas extracciones, 1 por hora, recogiendo de 0,14 a 0,21 I por extracción. A continuación los tiempos de recogida se han ido espaciando, como en el caso anterior, efectuando 4 extracciones cada 24 horas durante 4 ciclos ( 1 extracción cada 3 horas y otra cada 15 horas). Posteriormente y hasta el final del ensayo, las extracciones se han efectuado cada 24 horas, durante 32 días, recogiendo 3 a 4 litros de agua desionizada cada 24 horas.

El volumen total de agua desionizada que ha atravesado el lecho ha sido 129,7 I durante 37 días.

\subsection{Evolución del contenido de $\mathrm{Ca}$ (II)}

Estudio de los valores analíticos.

En la figura 3 se han representado en abscisas las extracciones (volumen acumulado, en I) y en ordenadas las concentraciones de $\mathrm{Ca}$ (II), en (moles/litro) $\times 10^{-2}$, presentes en cada una de las extracciones de agua desionizada, que ha atravesado el lecho de cemento hidratado durante 7 y 28 días.

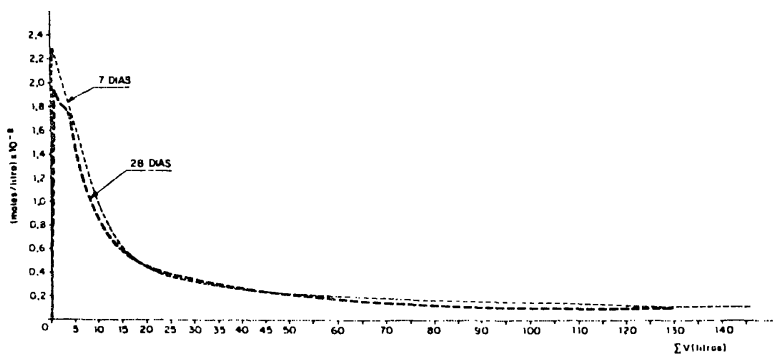

Fig. 3.-Sistemas: cemento 1 hidratado (7 y 28 dias)-agua desionizada. Evolución del contenido de ion $\mathrm{Ca}$ (II)

En la primera extracción $(0,18$ l) del lecho de cemento hidratado y curado durante 7 dias, la concentración de Ca (II) es $1,4 \times 10^{-2}$ moles/litro y en la segunda $(0,16 \mathrm{l} / \mathrm{h})$, se alcanza el valor máximo $2,3 \times 10^{-2}$ moles/l. A continuación, la cantidad de $\mathrm{Ca}$ (II) extraída experimenta un descenso exponencial; cuando han pasado 40 I y hasta que han atravesado el lecho 90 I, la cantidad por extracción ( 24 horas) es, aproximadamente, $0,2 \times 10^{-2}$ moles/litro.

Desde esta extracción y hasta el final (144,4 I) la cantidad de $\mathrm{Ca}$ (II) es, por extracción, aproximadamente $0,1 \times 10^{-2}$ moles/litro.
En el lecho de cemento hidratado, curado durante 28 días, las cantidades de $\mathrm{Ca}$ (II) extraídas de las primeras 20 extracciones (17,8 I), es inferior al caso del lecho correspondiente al cemento hidratado y curado durante 7 días y del mismo orden en los restantes. La evolución de las cantidades de $\mathrm{Ca}$ (II), por extracción, es análoga en ambos casos (fig. 3).

En la figura 4 se han representado en abscisas las extracciones acumulativas (volumen total de agua desionizada que ha pasado por cada uno de los lechos, cemento hidratado y curado durante 7 y 28 días) y en ordenadas los moles totales de $\mathrm{Ca}$ (II) extraídos.

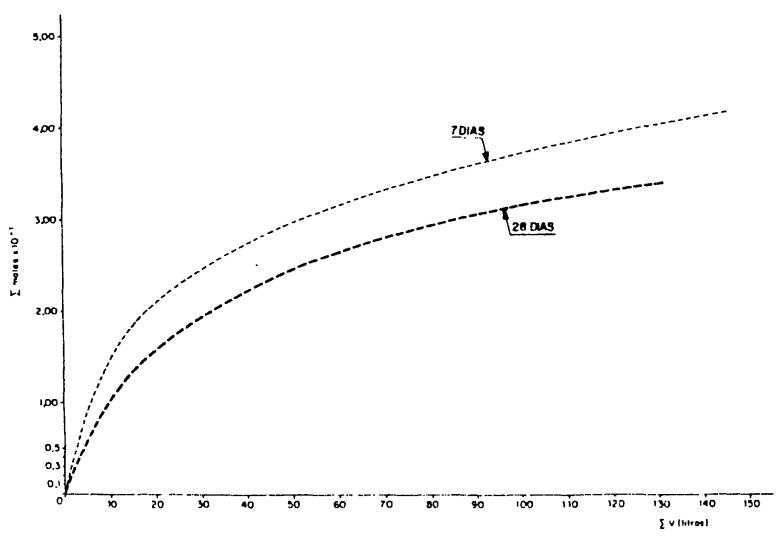

Fig. 4.-Sistemas: cemento 1 hidratado (7 y 28 días)-agua desionizada. Evolución del contenido de ion $\mathrm{Ca}$ (II) extraído, total.

La cantidad de Ca (II) extraída de ambos lechos de cemento es función del volúmen de agua desionizada que ha atravesado el lecho; esta cantidad aumenta rápidamente en las primeras extracciones y, a continuación, dicho aumento, se hace menor hasta llegar a ser prácticamente constante, sobre todo después que han atravesado el lecho 65 । de agua desionizada. Dicha cantidad de Ca (II) extraida es mayor en el caso del lecho de cemento hidratado y curado durante 7 días.

\section{Balance iónico}

En la tabla 1 figuran los contenidos de $\mathrm{Ca}$ (II), en moles, que existen en los $53,49 \mathrm{~g}$ de cemento puesto inicialmente en el lecho y en los $23,76 \mathrm{~g}$ de cemento que quedan al final, una vez que han pasado a su través 144,4 I de agua desionizada; dichas cantidades están referidas al producto calcinado. Así mismo, se encuentran los contenidos de dicho ion en el agua desionizada antes y después de pasar por el lecho de cemento hidratado y granulado, del sistema cemento 1 hidratado (7 días) agua desionizada. 
TABLA 1

Sistema: cemento 1 hidratado (7 dias)-agua desionizada.

Cantidades de Ca (II), en moles, en los componentes (iniciales y finales).

\begin{tabular}{|l|c|c|c|c|}
\hline \multirow{2}{*}{ Componentes } & \multicolumn{2}{|c|}{ Cantidades } & \multicolumn{2}{c|}{ Ca (II), moles } \\
\cline { 2 - 5 } & iniciales & finales & iniciales & finales \\
\hline Cemento & $53,49 \mathrm{~g}$ & $23,76 \mathrm{~g}$ & 0,57 & 0,16 \\
Agua desionizada & $144,4 \mathrm{I}$ & $144,4 \mathrm{I}$ & 0,00 & 0,42 \\
\hline Suma & & & 0,57 & 0,58 \\
\hline
\end{tabular}

En la tabla 2 se incluyen los contenidos del mencionado ion $\mathrm{Ca}$ (II) en los $53,49 \mathrm{~g}$ y 24,23 $\mathrm{g}$ de cemento del lecho, referidos al producto calcinado, antes y después de pasar 129,7 I de agua desionizada por el lecho de cemento hidratado y granulado del sistema cemento 1 hidratado (28 dias)-agua desionizada.

TABLA 2

Sistema: cemento 1 hidratado

(28 dias)-agua desionizada.

Cantidades de Ca (II), en moles, en los componentes (iniciales y finales).

\begin{tabular}{|l|c|c|c|c|}
\hline \multirow{2}{*}{ Componentes } & \multicolumn{2}{|c|}{ Cantidades } & \multicolumn{2}{c|}{ Ca (II), moles } \\
\cline { 2 - 5 } & iniciales & finales & iniciales & finales \\
\hline Cemento & $53,49 \mathrm{~g}$ & $24,23 \mathrm{~g}$ & 0.57 & 0,18 \\
Agua desionizada & $129,7 \mathrm{I}$ & $129,7 \mathrm{i}$ & 0,00 & 0,34 \\
\hline Suma & & 0,57 & 0.52 \\
\hline
\end{tabular}

En ambas tablas se puede observar que, aproximadamente, el $72 \%$ del ion $\mathrm{Ca}$ (II) presente en el lecho de cemento $(0,57$ moles) se encuentra en el conjunto de las distintas fracciones $(144,4$ I) de agua desionizada que han atravesado el lecho de cemento (0,42 moles) del primer sistema, mientras que en el segundo sistema, que corresponde al cemento hidratado y conservado durante 28 días en la cámara húmeda, el contenido de $\mathrm{Ca}$ (II) en el conjunto de las fracciones de agua desionizada que han pasado por el lecho $(129,7$ I) es 0,34 moles $<>68,4 \%$ del total. El resto de dicho ion ha quedado en los compuestos correspondientes de ambos lechos $(0,16$ moles $<>28 \%$ del inicial, en el primer sistema, y 0,18 moles $<>31,6 \%$, en el segundo sistema).

Las cantidades de cemento, en ambos casos, han experimentado una disminución análoga
$(29,73 \mathrm{~g}<>55,58 \%$, en el primer sistema y $29,26 \mathrm{~g}\langle>54,70 \%$, en el segundo), que se corresponden fundamentalmente con las de Ca (II) puestas en juego.

\subsection{Evolución del contenido de $\mathrm{SO}_{4}$ (II)}

\section{Estudio de los valores analíticos}

En la figura 5 se han representado en abscisas las extracciones (volumen acumulado en I) y en ordenadas las concentraciones de $\mathrm{SO}_{4}$ (II), en (moles/litro) $\times 10^{-4}$, presentes en cada una de las 24 primeras extracciones, cuando 30,15 I de agua desionizada han atravesado el lecho de cemento hidratado y curado durante 7 dias, $y$ de las 26 extracciones, que corresponden a 29,00 I, para el caso del cemento hidratado y curado durante 28 días. En las restantes extracciones efectuadas, en ambos casos, no ha sido posible determinar gravimétricamente el contenido de $\mathrm{SO}_{4}$ (II), debido a la pequeña concentración existente.

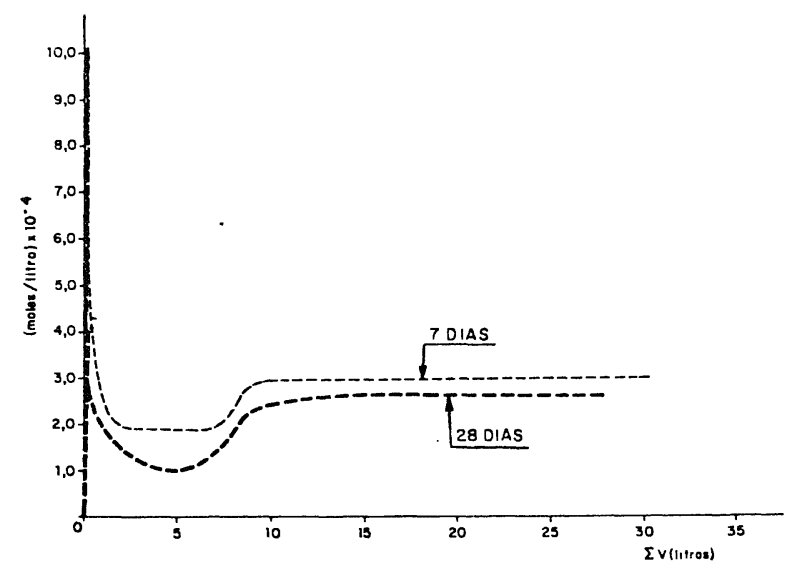

Fig. 5.-Sistemas: cemento 1 hidratado ( 7 y 28 días)-agua desionizada. Evolución del contenido de ion $\mathrm{SO}_{4}$ (II).

La concentración de $\mathrm{SO}_{4}$ (II) en la primera extracción $(0,18 \mathrm{I})$ del lecho de cemento hidratado y curado durante 7 días es $10,0 \times 10^{-4}$ moles/litro, valor máximo, que disminuye brusca y exponencialmente hasta tomar el valor $2,0 \times 10^{-4}$ moles/litro cuando han pasado 8,00 । de agua desionizada y posteriormente el valor $3,0 \times 10^{-4}$ moles, valor que se mantiene prácticamente constante hasta la extracción que corresponde a 30,15 litros.

La concentración de $\mathrm{SO}_{4}$ (II) en la primera extracción $(0,211)$ del lecho de cemento hidratado y curado durante 28 días es $4,0 \times 10^{-4}$ moles/litro; en la siguiente extracción, toma el valor $1,0 \times 10^{-4}$ moles/litro que se mantiene prácticamente constante hasta que han atravesado el lecho 7,32 I de agua desionizada, para aumentar en la siguiente extracción $\left(8,01\right.$ l) alcanzando el valor $2,5 \times 10^{-4}$ moles 
litro, que se mantiene de nuevo prácticamente constante hasta que han atravesado el lecho 29,05 I.

Las cantidades extraídas de $\mathrm{SO}_{4}$ (II), en el caso del lecho de cemento hidratado y curado durante 28 días, son inferiores que las correspondientes al lecho de cemento hidratado y curado durante menor número de días (7 días).

En la figura 6 se han representado en abscisas las extracciones acumulativas (volumen total de agua desionizada que ha pasado por cada uno de los lechos) y en ordenadas los moles $X$ $10^{-3}$ totales de $\mathrm{SO}_{4}$ (II) extraídos, en donde puede apreciarse que los mencionados moles $\times 10^{-3}$ extraídos de $\mathrm{SO}_{4}$ (II) son función del volumen de agua desionizada que ha atravesado el lecho y del tiempo de curado del cemento; mayor el correspondiente a 7 días.

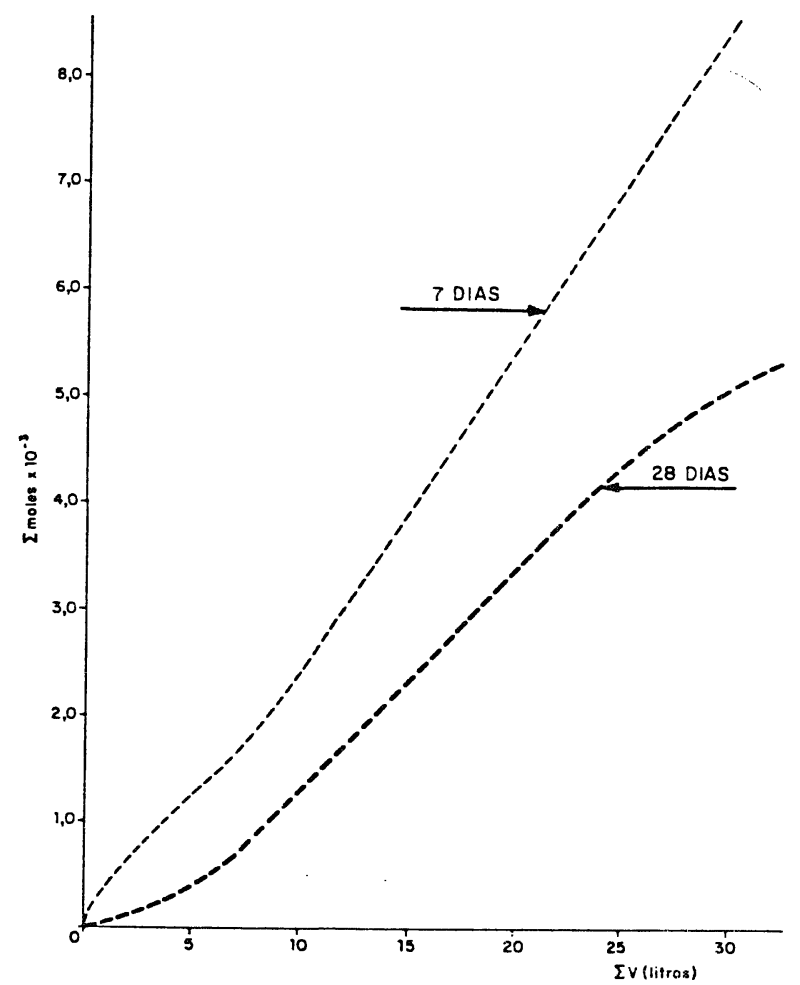

Fig. 6.-Sistemas: cemento 1 hidratado (7 y 28 días)-agua desionizada. Evolución del contenido de ion $\mathrm{SO}_{4}$ (II) extraido, total.

\section{Balance iónico}

En la tabla 3 figuran los contenidos de $\mathrm{SO}_{4}$ (II), en moles, que existen en los $53,49 \mathrm{~g}$ de cemento puestos incialmente en el lecho y en los 23,76 g de cemento que existen después de pasar a su través 144,4 I de agua desionizada; ambas cantidades de cemento están referidas a los productos calcinados. Asi mismo, se encuentran en dicha tabla los contenidos de $\mathrm{SO}_{4}$ (II) en el agua desionizada antes de pasar por el lecho y en el conjunto de las fracciones que corresponden a los primeros 60,25 I de agua desionizada, en donde se han podido determinar, una vez que atravesaron el mencionado lecho de cemento hidratado y granulado del sistema cemento 1 hidratado ( 7 días)-agua desionizada.

\section{TABLA 3}

Sistema: cemento 1 hidratado

(7 dias)-agua desionizada.

Cantidades de $\mathrm{SO}_{4}(\mathrm{II})$, en moles, en los componentes (iniciales y finales).

\begin{tabular}{|l|c|c|c|c|}
\hline \multirow{2}{*}{ Componentes } & \multicolumn{2}{|c|}{ Cantidades } & \multicolumn{2}{c|}{ SO $_{4}$ (II), moles } \\
\cline { 2 - 5 } & iniciales & finales & iniciales & finales \\
\hline Cemento & $53,49 \mathrm{~g}$ & $23,76 \mathrm{~g}$ & $2,65 \times 10^{-2}$ & $0,90 \times 10^{-2}$ \\
Agua desionizada & $144,4 \mathrm{I}$ & $144,4 \mathrm{I}$ & 0,00 & $1,61 \times 10^{-2}$ \\
\hline Suma & & $2,65 \times 10^{-2}$ & $2,51 \times 10^{-2}$ \\
\hline
\end{tabular}

En la tabla 4 se incluyen los contenidos de dicho ion $\mathrm{SO}_{4}$ (II) en los $53,49 \mathrm{~g}$ y $24,23 \mathrm{~g}$ de cemento del lecho, referidos al producto calcinado, antes y después de pasar 129,7 | de agua desionizada; así mismo, se incluyen las cantidades que existen de $\mathrm{SO}_{4}$ (II) en el agua desionizada antes de pasar por el lecho y en las fracciones primeras en donde se han podido determinar, que hacen un total de 56,75 I, una vez que atravesaron el lecho de cemento de sistema cemento 2 hidratado (28 dias)agua desionizada.

\section{TABLA 4}

Sistema: cemento 1 hidratado

(28 días)-agua desionizada.

Cantidades de $\mathrm{SO}_{4}(\mathrm{II})$, en moles, en los componentes (iniciales y finales).

\begin{tabular}{|l|c|c|c|c|}
\hline \multirow{2}{*}{ Componentes } & \multicolumn{2}{|c|}{ Cantidades } & \multicolumn{2}{c|}{$\mathrm{SO}_{4}$ (II), moles } \\
\cline { 2 - 5 } & iniciales & finales & iniciales & finales \\
\hline Cemento & $\begin{array}{c}5,49 \mathrm{~g} \\
129,7 \mathrm{I}\end{array}$ & $\begin{array}{c}24,23 \mathrm{~g} \\
129,7 \mathrm{I}\end{array}$ & $\begin{array}{c}2,65 \times 10^{-2} \\
0,00\end{array}$ & $\begin{array}{c}1,04 \times 10^{-2} \\
0,98 \times 10^{-2}\end{array}$ \\
\hline Agua desionizada & $12,08 \times 10^{-2}$ & $2,02 \times 10^{-2}$ \\
\hline Suma & & & $2,65 \times 10^{-2}$ & \\
\hline
\end{tabular}

En ambas tablas se puede observar que en el primer sistema (tabla 3) han quedado en el lecho $0,90 \times 10^{-2}$ moles $(33,96 \%)$ de los $2,65 \times 10^{-2}$ moles iniciales, una vez que han pasado 144,4 litros de agua desionizada y que 
en el segundo sistema (tabla 4) han quedado $1,04 \times 10^{-2}$ moles $(39,4 \%)$ de los $2,65 \times 10^{-2}$ moles originales cuando han pasado $129,7 \mathrm{li}$ tros de agua desionizada.

\subsection{Evolución del pH y de la conducti- vidad}

En la figura 7 se han representado los valores del $\mathrm{pH}$ y de la conductividad (mho/cm), en ordenadas, en función del volumen de agua desionizada (litros) que ha atravesado el lecho de cemento hidratado y granulado, en abscisas.

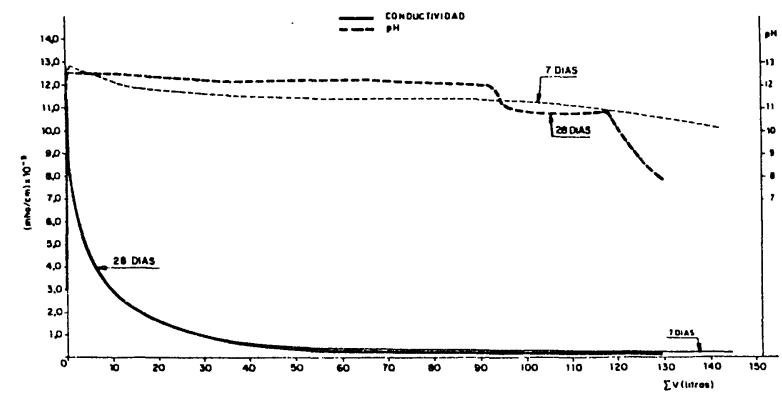

Fig. 7.-Sistemas: cemento 1 hidratado (7 y 28 días)-agua desionizada. Evolución del $\mathrm{pH}$ y de la conductividad.

\section{Evolución del pH}

En ambos sistemas, cemento 1 hidratado ( 7 días)-agua desionizada y cemento 1 hidratado (28 dias)-agua desionizada, el $\mathrm{pH}$ sigue una evolución análoga. En las extracciones correspondientes a los primeros cinco litros, se obtienen los valores mayores comprendidos entre 12,3 y 12,8 ; a continuación, el pH desciende por debajo de 12,0 y sigue disminuyendo, para el caso del cemento hidratado y curado durante 7 días, si bien se mantiene prácticamente constante 12,0 para el caso del cemento hidratado y curado durante 28 días hasta que han atravesado el lecho 117 y 93,5 I, respectivamente. A partir de este momento, en el primer sistema el $\mathrm{pH}$ toma valores comprendidos entre 11,0 y 9,5 y en el segundo entre 11,9 y 7,8

\section{Evolución de la conductividad}

La conductividad del agua desionizada, que ha atravesado los lechos de cemento 1 de ambos sistemas, alcanza los valores mayores $(13,1 \times$ $10^{-3}$ y $11,3 \times \times 10^{-3} \mathrm{mho} / \mathrm{cm}$ para ambos sistemas) cuando han atravesado las primeras cantidades de agua desionizada $(0,18$ y 0,21 litros, respectivamente); a continuación, disminuye exponencialmente, alcanzando el valor $0,1 \times 10^{-3} \mathrm{mho} / \mathrm{cm}$ cuando han atravesado el lecho 113 y 122,5 I, respectivamente, de agua desionizada, valor que se mantiene hasta el final de ambos ensayos.

\subsection{Estudio del cemento hidratado e hidratado-atacado de los lechos por DRX}

En la figura 8 se incluyen los DRX del cemento 1 hidratado ( 7 dias) y cemento 1 hidratado (28 día) antes y después de someterlos a la acción del agua desionizada, en los cuales se han detectado los picos de los siguientes compuestos cristalinos.
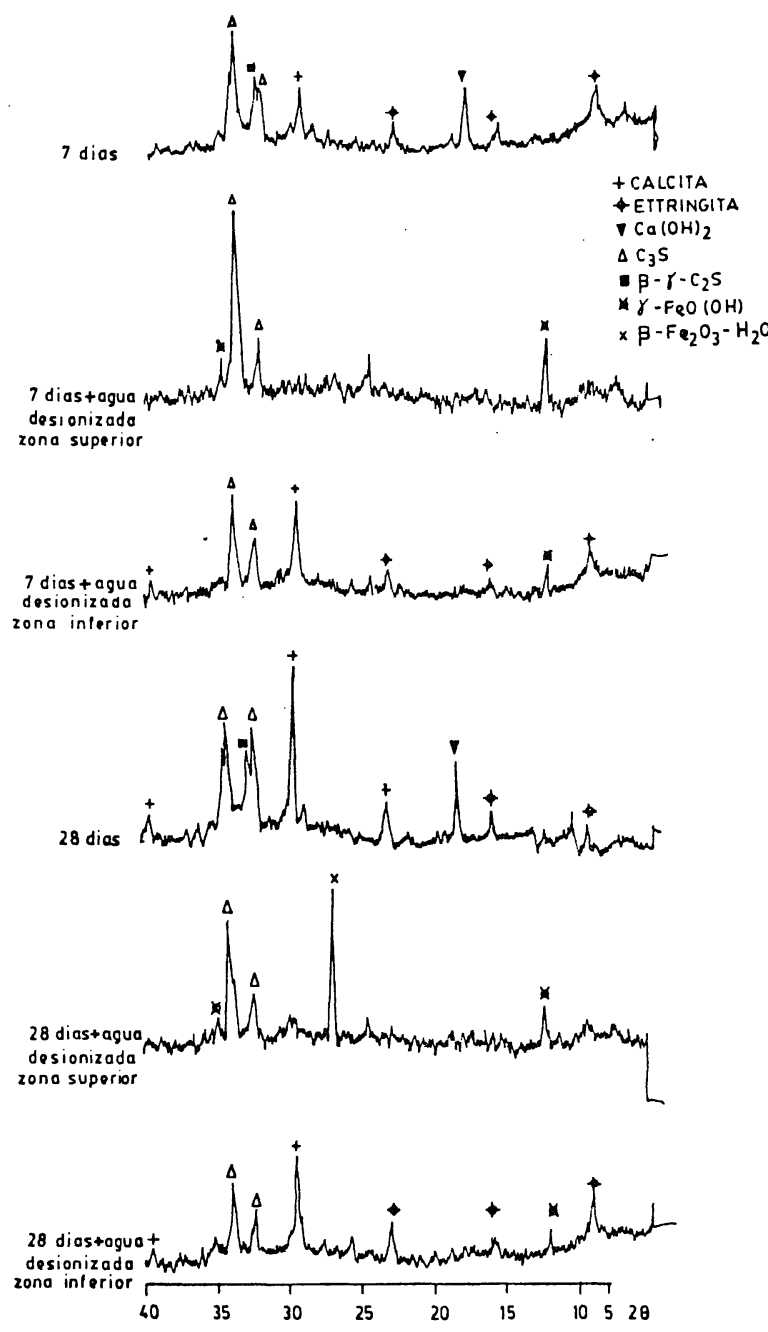

Fig. 8.-Sistemas: cemento 1 hidratado (7 y 28 dias)-agua desionizada. DRX de los cementos hidratados e hidratados-atacados.

* Diagramas de difracción de rayos $X$ del cemento 1 hidratado ( 7 y 28 días)

- Ettringita; los picos de este compuesto tienen una intensidad análoga en ambos DRX. 
- Portlandita; la intensidad de los picos en el DRX del cemento hidratado y curado durante 28 días es 1,25 veces mayor que la del DRX del cemento hidratado y curado durante 7 días, como consecuencia del avance de las reacciones de hidratación.

- Calcita; la intensidad de los picos en el DRX del cemento hidratado y curado durante 28 días es 3 veces mayor que la del DRX del cemento hidratado y curado 7 días.

- Compuestos anhidros del cemento, cuyos picos presentan intensidad análoga en los dos diagramas.

* Diagramas de difracción de rayos $X$ del cemento 1 hidratado ( 7 y 28 dias), sometido a la acción del agua desionizada.

Ambos lechos se han dividido en dos partes, por presentar aspectos organolépticos diferentes.

En el DRX de la muestra correspondiente a la parte superior del lecho del cemento hidratado y curado durante 7 días sólo se han detectado los picos de los compuestos anhidros del cemento y un compuesto de hierro, que responde a la fórmula $\gamma-\mathrm{FeO}(\mathrm{OH})$. En el $\mathrm{DRX}$ que corresponde a la muestra tomada de la parte inferior se han detectado los picos de los siguientes compuestos cristalinos:

- Ettringita, cuyos picos son de intensidad sensiblemente análoga a los de la muestra sin atacar.

- Calcita, cuya intensidad es 1,5 veces mayor.

- Compuestos anhidros del cemento.

Los picos de la portlandita han desaparecido totalmente.

En el DRX de la parte superior del lecho de cemento hidratado y curado durante 28 días se han detectado los picos de los compuestos, anhidros del cemento y en esta ocasión dos compuestos de hierro, que responden a las fórmulas $\gamma-\mathrm{FeO}(\mathrm{OH})$ y $\beta-\mathrm{Fe}_{2} \mathrm{O}_{3} \cdot \mathrm{H}_{2} \mathrm{O}$ y en el de la parte inferior los picos de los siguientes compuestos cristalinos:

- Ettringita, con intensidad ligeramente inferior a la de la muestra sin atacar.

- Calcita, cuya intensidad es aproximadamente la mitad.

- Compuestos anhidros, con intensidad menor.
Los picos de la portlandita, al igual que para el cemento hidratado y curado durante 7 dias, han desaparecido.

\section{INTERPRETACION DE RESULTADOS}

Los iones $\mathrm{Ca}$ (II) que aparecen en el agua desionizada, que ha atravesado los lechos de cemento hidratado y granulado, poceden fundamentalmente de la disolución del $\mathrm{Ca}(\mathrm{OH})_{2}$ formado durante las reacciones de hidratación del $\mathrm{C}_{3} \mathrm{~S}$ y $\mathrm{C}_{2} \mathrm{~S}$ de la fracción clínker del cemento, así como a partir del $\mathrm{CaO}$ libre, según la reacción $(A)$ :

(A)

$$
\begin{gathered}
\mathrm{Ca}(\mathrm{OH})_{2} \text {. sólido }+\mathrm{H}_{2} \mathrm{O} \rightleftharpoons \\
\rightleftharpoons \mathrm{Ca}(\mathrm{OH})_{2} \text {.disuelto } \rightleftharpoons \mathrm{Ca}(\mathrm{II})+2 \mathrm{OH}(\mathrm{I})
\end{gathered}
$$

En el sistema cemento hidratado (7 dias)-agua desionizada se alcanza la cantidad correspondiente a la solubilidad teórica en la segunda extracción, cuando han pasado $0,16 \mid(2,3 \times$ $\times 10^{-2}$ moles/litro); a cotinuación, dicha cantidad disminuye exponencialmente, llegando a ser 23 veces menor $\left(0,1 \times 10^{-2}\right.$ moles/litro $)$ en las extracciones superiores al paso de $90 \mathrm{li}$ tros de agua desionizada. En el sistema cemento 2 hidratado (28 días)-agua desionizada las cantidades extraídas son menores, no llegando a alcanzarse la correspondiente a la solubilidad teórica como consecuencia del avance de las reacciones de hidratación $y$, especialmente, de la mayor carbonatación que, a pesar de las precauciones tomadas, ha experimentado la pasta de cemento curada durante mayor tiempo, como se ha probado por DRX.

Los silicatos de calcio hidratados son poco solubles en agua, $50 \mathrm{mg} / \mathrm{l}$ como $\mathrm{CaO}(14)(15)<>$ $<>66 \mathrm{mg} / \mathrm{l}$ como $\mathrm{Ca}(\mathrm{OH})_{2}$.

La ettringita se disuelve ligeramente con separación de gel de alúmina, sulfato de calcio dihidratado e hidróxido de calcio, según ciertos autores (16) (17), y es extraordinariamente poco soluble $\left(0,001 \mathrm{~g}\right.$ de $\mathrm{SO}_{3}$ por $100 \mathrm{ml}$ de agua saturada de cal), según otros (18).

En este trabajo se ha probado por DRX que en los diagramas del cemento hidratado de los lechos sometidos a la acción del agua desionizada -tanto los de la zona superior de la columna como los de la zona inferior- han desaparecido los picos del hidróxido de calcio y se ha producido una disminución de la intensidad de los picos de los compuestos anhidros, como consecuencia del avance de las reacciones de hidratación al producirse, de un modo especial, la eliminación del mencionado hidróxido.

La intensidad de los picos de la ettringita de 
los DRX de las zonas inferiores de la columna de los lechos es sensiblemente análoga, o ligeramente inferior, a los correspondientes a las muestras de cemento hidratado sin someter a la acción del agua desionizada. Sin embargo, en los diagramas de las zonas superiores han desaparecido dichos picos. El ion $\mathrm{SO}_{4}$ (II) se ha encontrado en dicha agua desionizada, una vez que ha atravesado los lechos de cemento; la disminución experimentada por el contenido de dicho ion en el cemento hidratado ha sido del $34 \%$ y $39,4 \%$, según se trate del cemento hidratado curado durante 7 días ó 28 días, respectivamente.

En los DRX del cemento hidratado sometido a la acción del agua desionizada se han detectado unos picos, que se han atribuido a los compuestos $\gamma-\mathrm{FeO}(\mathrm{OH})$ y $\beta-\mathrm{Fe}_{2} \mathrm{O}_{3} \cdot \mathrm{H}_{2} \mathrm{O}$, en el DRX de la zona superior del cemento hidratado y curado durante 28 días; compuestos que pueden proceder de la descomposición de los aluminatoferritos del cemento, hecho que se piensa comprobar.

$\mathrm{El} \mathrm{pH}$ de las distintas fracciones extraídas de agua desionizada viene regido por el equilibrio A. En la primera extracción, para ambos sistemas, se observa un incremento brusco, prácticamente de 6 unidades con relación al del agua desionizada, llegando a alcanzar valores comprendidos entre 12,3 y 12,8 .

A medida que la concentración de iones $\mathrm{OH}(\mathrm{I})$ disminuye, por disolverse menores cantidades de $\mathrm{Ca}(\mathrm{OH})_{2}$, lo hace también el valor del $\mathrm{pH}$, manteniéndose prácticamente constante (próximo a 12) hasta que han pasado por el lecho 117 y 93,5 litros de agua desionizada. A partir de estas etapas, el $\mathrm{pH}$ toma valores comprendidos entre 11,0 y 9,5 , para el primer sistema, y entre 11,9 y 7,8 para el segundo.

La conductividad del agua desionizada, que ha atravesado los lechos de cemento, es función de la cantidad de iones presentes en dicha agua desionizada. El valor de la conductividad pasa de $0,04 \times 10^{-8} \mathrm{mho} / \mathrm{cm}$, valor del agua desionizada, a $13,1 \times 10^{-3}$ y $11,3 \times 10^{-3}$ $\mathrm{mho} / \mathrm{cm}$ en las fracciones del agua de la primera extracción $(0,18$ y 0,21 litros respectivamente) de ambos sistemas; a continuación disminuye exponencialmente, del mismo modo que lo hacen las cantidades de $\mathrm{Ca}$ (II).

Teniendo en cuenta que las conductividades iónicas, a dilución infinita y a $25^{\circ} \mathrm{C}$, expresada en ohm ${ }^{-1} . \mathrm{cm}^{2}$.equiv ${ }^{-1}$ de los iones $\mathrm{Ca}$ (II), $\mathrm{OH}$ (I) y $\mathrm{SO}_{4}$ (II) son $59,5,198,0$ y 80,0 (19), respectivamente, se considera que la conductividad en los sistemas estudiados, del mismo modo que la del $\mathrm{pH}$, viene regida de un modo especial por el equilibrio (A), disminuyendo conforme lo hacen las concentraciones de los iones $\mathrm{Ca}$ (II) y $\mathrm{OH}(1)$, al disolverse menor cantidad de $\mathrm{Ca}(\mathrm{OH})_{2}$. La disminución de la conductividad se acusa más intensamente que la del pH.

La disolución progresiva del $\mathrm{Ca}(\mathrm{OH})_{2}$, electrolito débil, en el agua desionizada que atraviesa los lechos de cemento, favorece las reacciones de hidratación de la fracción clínker del cemento, liberando más portlandita que pasa al agua, en forma iónica, llegando a desaparecer del lecho de cemento, a la vez que los silicatos de calcio anhidros se degradan. Esta eliminación del hidróxido de calcio es la causa fundamental de la pérdida de masa que han experimentado los mencionados lechos de cemento, y en menor grado la correspondiente a la disolución de los compuestos a base de sulfatos; en efecto, la pérdida de masa en ambos sistemas ha sido del orden de $29 \mathrm{~g}$, que se corresponden con la eliminación de 0,41 moles (primer sistema) y 0,40 moles (segundo sistema) de $\mathrm{Ca}$ (II) $[0,57$ moles iniciales - 0,16 a 0,17 moles finales] $\langle>0,41$ a 0,40 moles de $\mathrm{Ca}(\mathrm{OH})_{2}<>30,3 \mathrm{~g}$ y $29,6 \mathrm{~g}$.

\section{CONCLUSIONES}

\section{Primera}

El hidróxido de calcio presente en los lechos fabricados con cemento 1 hidratado y granulado, que procede de las reacciones de hidratación de la fracción clínker, se disuelve en el agua desionizada que atraviesa dichos lechos; asimismo, los compuestos a base de sulfatos, $y$ de un modo especial la ettringita, se disuelven parcialmente.

\section{Segunda}

La cantidad de $\mathrm{Ca}$ (II) en el agua desionizada extraída de los lechos de cemento hidratado y granulado, que procede de los compuestos de calcio del cemento, es función del tiempo de curado del cemento hidratado y del volumen de dicha agua desionizada que ha pasado por el lecho, experimentado una disminución exponencial conforme aumenta el mencionado volumen de agua desionizada.

El cemento hidratado de los lechos, sometido a la acción del agua desionizada, experimenta una disminución considerable de masa, que llega a ser superior al $50 \%$.

\section{Tercera}

La conductividad de las distintas fracciones recogidas de agua desionizada, que han atravesado los lechos de cemento, experimenta un incremento brusco en las dos primeras extracciones $y$, a continuación, una disminución ex- 
ponencial conforme aumenta el volumen de agua desionizada. La evolución de la conductividad, a lo largo de las distintas extracciones de agua desionizada, es función, principalmente, de la concentración de $\mathrm{OH}$ (I) en la disolución que, a su vez, depende de la concentración de $\mathrm{Ca}(\mathrm{OH})_{2}$ disuelto.

Así mismo, el valor de $\mathrm{pH}$ de las mencionadas fracciones de agua desionizada es función de la cantidad de $\mathrm{Ca}(\mathrm{OH})_{2}$ disuelto; los valores máximos $(\mathrm{pH}=12,3$ a 12,8$)$ se obtienen en las dos primeras extracciones y los menores $(\mathrm{pH}=$ $=12$ a 7,8 , según los sistemas) en las últimas.

\section{Cuarta}

Los compuestos cristalinos del cemento hidra- tado (de un modo especial ettringita, portlandita y calcita) y de la fracción clínker anhidro, que se han sometido a la acción del agua desionizada, experimentan modificaciones considerables. Así, en los DRX obtenidos, se observa que han desaparecido los picos de la portlandita y que ha disminuído la intensidad de los picos de la ettringita, así como la de los silicatos anhidros de la fracción clínker al avanzar las reacciones de hidratación.

Además, se han detectado en determinadas zonas de los lechos de cemento los picos de los compuestos cristalinos $\gamma$ - $\mathrm{FeO}(\mathrm{OH})$ y $\beta$ $\mathrm{Fe}_{2} \mathrm{O}_{3} \cdot \mathrm{H}_{2} \mathrm{O}$, que proceden de la descomposición de los aluminato-ferritos correspondientes.

\section{BIBLIOGRAFIA}

(1) SAGRERA-MORENO, J L y GASPAR-TEBAR, D. (1980): Resistencia química del hormigón. IV. Acción del agua de mar: influencia de la adición de escoria a un cemento portland de alta resistencia inicial. Evolución de las resistencias mecánicas a flexotracción y del coeficiente de corrosión. Materiales de Construcción, abril-mayo-junio, $178,17-38$

(2) GASPAR-TEBAR, D. y SAGRERA-MORENO, J. L. (1981): Resistencia quimica del hormigón. IX. Influencia de la adición de escoria a un cemento portland de alta resistencia inicial. Estudio por DRX del sistema: cemento 1/escoria-agua potable filtrada. Materiales de Construcción. 181, 33-44.

(3) SAGRERA-MORENO, J. L. y GASPAR-TEBAR, D. (1982): Resistencia química del hormigón. XV. Acción de una disolución saturada de yeso: influencia de la adición de escoria a un cemento portland de alta resistencia inicial. Evolución de las resistencias mecánicas a flexotracción y de los coeficientes de corrosión. Materiales de Construcción, 185, 29-42.

(4) GASPAR-TEBAR, D. y SAGRERA-MORENO, J. L. (1982): Resistencia química del hormigón. XVI. Acción del agua de mar: influencia de la adición de escoria a un cemento portland de alta resistencia inicial. Estudio por DRX. Materiales de Construcción, 186, 39-60.

(5) SAGRERA-MORENO, J. L. y GASPAR-TEBAR, D. (1983): Resistencia química del hormigón. XXI. Influencia de la adición de escoria a un cemento portland de alta resistencia inicial. Estudio de la concentración iónica del sistema cemento 1/escoria-agua potable filtrada. Materiales de Construcción, 192, 33-41.

6) GASPAR-TEBAR, D. y SAGRERA-MORENO J. L (1984): Resistencia química del hormigón XXII Influencia de la adición de escoria a un cemento portland de alta resistencia inicial. Estudio de la concentración iónica del sistema: cemento 1/escoria-agua de mar artificial. Materiales de Construcción, 193, 51-68.

(7) DIAZ-MORA, R., SAGRERA-MORENO, J. L. y GASPAR-TEBAR, D. (1980): Resistencia química del hormigón. VI. Acción del agua de mar artificial que fluye a través de un lecho de mortero fabricado con un cemento portland de alta resistencia inicial. Materiales de Construcción, 179, 81-99.

(8) GASPAR-TEBAR, D., SAGRERA-MORENO, J. L. y BERMEJO-MUÑOZ, M. ${ }^{a}$ F. (1981): Resistencia química del hormigón. XI. Contribución al estudio del sistema: cemento P-450-Y hidratado-agua desionizada. Materiales de Construcción, 183, 17-30.

(9) BERMEJO-MUÑOZ, M. a F., SAGRERA-MORENO, J. L. y GASPAR-TEBAR, D. (1981): Resistencia quimica del hormigón. XIII. Contribución al estudio del sistema: cemento P-450-Y hidratado-disolución de sulfato de sodio. Materiales de Construcción, 184, 51-63.

(10) GASPAR-TEBAR, D., SAGRERA-MORENO, J. L. y BERMEJO-MUÑOZ, M. ${ }^{a}$ F. (1981): Resistencia química del hormigón. XIV. Contribución al estudio del sistema: cemento P-450-Y hidratado-disolución de sulfato de magnesio. Materiales de Construcción, 184, 65-82.

(11) Pliego de Prescripciones Técnicas Generales para la Recepción de Cemento, 1975. (RC-75): MOP, Servicio de Publicaciones. Madrid, 1975

(12) BICZOK, I. (1972): Corrosión y Protección del Hormigón. Ed. Urmo, Bilbao, pág. 211.

(13) DURIEZ, M. et ARRAMBADI, J. (1961): Nouv. Trait. Mat. Const., Tome 1. Ed. Dunod, París, págs. 439-440.

(14) ALEGRE, R. (1978): Comportement des ciments en milieu agressif. Annales de l'Inst. Techn. du Bât. et des Trav. Publ.; serie: Sols et Fondations, n. ${ }^{\circ} 159,364,78$.

(15) DREYFUS, J. (1960): La Chimie des Ciments. Edit. Eyrolles; Paris, Tome I, pág. 41. 
(16) TAYLOR, H. F. W. (1964): The Chemistry of Cements. Acad. Pres. Inc.; London and New York, vol. 1, pág. 261.

(17) LEA, F. M. y DESCH, E. H. (1960): Química del Cemento y de Hormigón. Tipografía Artística; Madrid, pág. 228.

(18) BOGUE, R. H. (1962): La Química del Cemento Portland. Edit. Dossat; Madrid, pág. 600.

(19) CHARLOT, G. (1963): Química Analítica General. Tomo II. Métodos electroquímicos y absorciométricos; cromatografía. Edt. Toray Masson, Barcelona, págs. 123-124.

\section{RECONOCIMIENTO}

Nuestro más sincero agradecimiento a las personas del Equipo de Durabilidad del IETcC: Amalia Rodríguez Pereira, Lucila López Solana, M.a Soledad Cid Fernández, Felipe Cantero Palacios y Manuel Cantero Palacios, así como a M.a Isabel Jiménez, por su valiosa colaboración en la realización de este trabajo.

\section{publicaciones del i.e.t.c.c.}

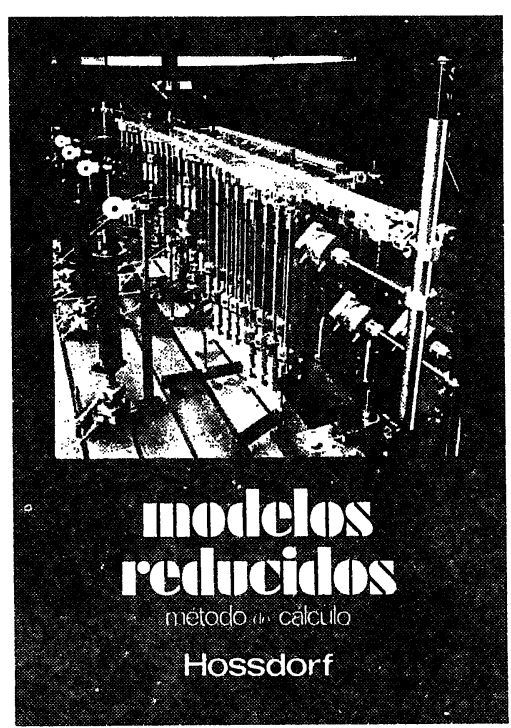

Modelos reducidos. Método de cálculo

H. Hossdorf, Ingeniero Civil

La técnica de los ensayos en modelos reducidos de estructuras sufre hoy dia una decisiva metamorfosis. Hasta hace poco era un medio más bien de artesania, que no siempre un media mas bien de aror academicos teorizantes para comprender el comportaque so acudio las mas de las veces, como a un que se acudib las mas de las veces, como a un cias. Sin embargo, en poco tiempo y gracias a su cias. Sin embargo, en poco tiempo y gracias a su conexión con los ordenadores dightales, se ha transque no puede quedar a un lado en la práctica diaria del Ingeniero Proyectista.

Un volumen encuadernado en cartoné plastificado 250 páginas, 158 figuras y fotografias.

Precios: 1.800 ptas.; \$ USA 26.00

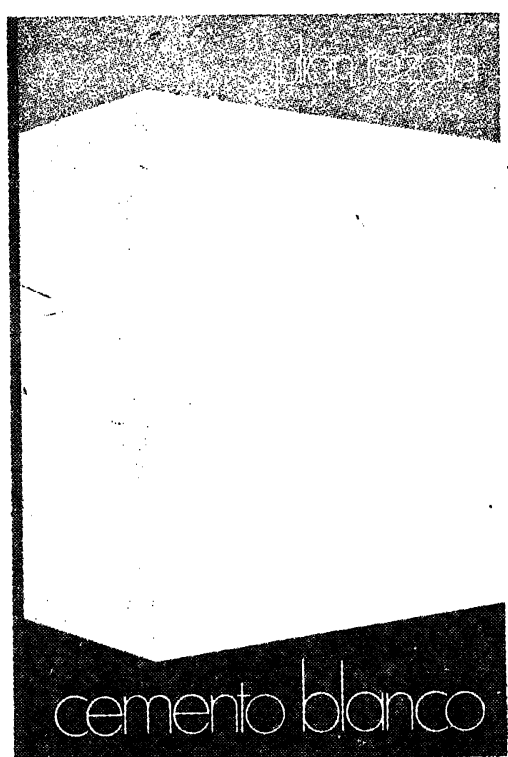

\section{Cemento blanco}

Julián Rezola

Ingeniero Quimico Dipl. I. Q. S.

Sabido es que existe una extensa y documentada bibliografia sobre el cemento gris: en cambio, no puede decirse lo mismo acerca del cemento portand blanco, ya que los escrios existentes se refin tan aquér.

El autor nos ofrece sus profundos conocimientos y su larga experiencia tanto en laboratorio como en fabricación.

La parte descriptiva del libro se complementa con gráficos, diagramas y fotografias de gran utilidad, propiada de

Un volumen encuadernado en cartoné policerado, de $17,4 \times 24,3 \mathrm{~cm}$, compuesto de 395 páginas. numerosas figuras, tablas y abacos.

Precios: España, 1.700 ptas.; extranjero, $\$ 24.00$

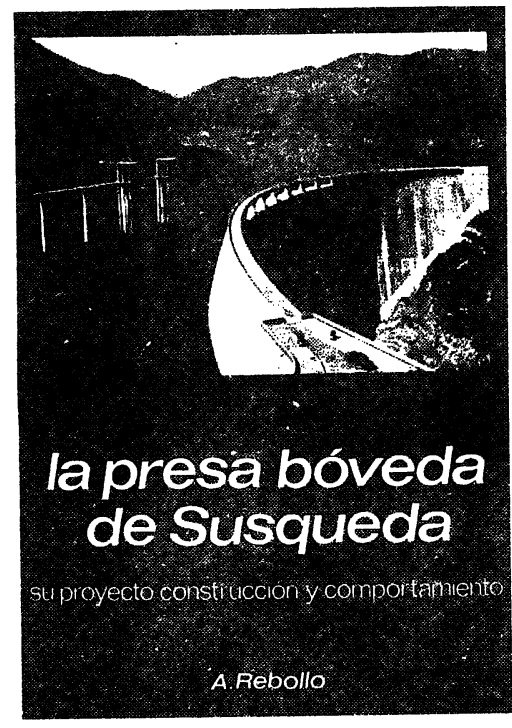

La presa bóveda de Susqueda

A. Rebollo,

Dr. Ingeniero de Caminos

El esfuerzo del constructor de presas se sitúa, por su pretension de perennidad, a contracorriente cor lo fungible. Pueden evocarse is 10.000 grandes presas en funcionamiento 0 en construcción grandes.pros lo que en gerontologicos para man inalienable pretensión de perennidad. En la medióa en que todas nuevas obras, grandes o pequeñas, son portadoras de riesgos ecológicos $y$, a veces, catastróficos, que riesgos ecologicos $y_{1}$ a veces, catastroficos, que
aumentan con el envejecimiento, la gerontologia de las presas es todo un emplazo. La acción adelantada de Arturo Rebollo en este terreno marca un camino a seguir para todos los que aman su propia obra con la devoción paternal que él ha puesto en Susqueda.

Un volumen encuadernado en cartoné plastificado con lomo de tela, de $18 \times 24,5 \mathrm{~cm}$, compuesto de 408 páginas, 330 figuras y fotografias y 39 tablas.

Precios: 1.700 ptas.; extranjero, \$ USA 24.00 . 